\title{
Optical Observations of Pulsars
}

\author{
Christian Gouiffes \\ Service d'Astrophysique, CEA/DSM/DAPNIA C.E. Saclay, 91191 \\ Gif-sur-Yuette Cedex-FRANCE \\ Hakki Ögelman \\ Department of Physics, University of Wisconsin-Madison, 1150 \\ University Avenue, Madison, WI53706 - USA
}

\section{Introduction}

In the course of a programme to study optical pulsars, we have observed at different occasions PSR0540-69, the $50 \mathrm{msec}$ pulsar in the LMC. Like the Crab pulsar, it has been detected at X-rays, optical and radio and its braking index $(\sim 2)$ determined. In this communication we report some results obtained on this pulsar, in particular the presence of narrow features in the pulse profile and compare it with a nearly simultaneous $\mathrm{X}$-ray observation.

\section{Observations and Analysis}

The observations were carried out at the $3.6 \mathrm{~m}$ telescope at La Silla observatory, Chile. The instrument consisted on a single channel photometer coupled with a photomultiplier (GaAs photocathode). The sampling frequency was $10 \mathrm{kHz}$ and the output of the photon counting system was recorded sequentially on magnetic tape. A set of filters and diaphragms were available. A standard analysis was then performed. After corrections to the solar system barycenter, the data were analyzed and the best period determined using the $Z_{2}^{2}$ test (Buccheri et al, 1983).

\section{Results and Discussion}

Fig. 1 shows the light curve of PSR0540-69 obtained in 1992, November 5 (exposure time $\sim 1$ hour, free light, seeing disc $\leq 1$ "). The period was measured to be $\mathrm{P}=50.405035$ (1) $\mathrm{msec}$ and phase 0 corresponds to $\mathrm{MJD}(\mathrm{TDB})=48931.33437451$. As already described (Seward et al 1984, Middleditch et al 1985, Gouiffes et al 1992) the pulsed profile consists of a broad peak with two narrow features on top of it separated by 0.25 in phase. Other narrow features are present in the pulse shape: one (phase $\sim 0.7$ and $\leq 1 \mathrm{msec}$ ) falls half a period before (or after) the centroid of the broad modulation. Other faint and narrow structures seem to appear in the falling trail of the peak. The presence of faint structures in the light curve has been recently noticed by a HST observation (Boyd et al, 1995). It be would interesting to get higher signal/noise light curve of PSR0540-69 to study in detail the behavior of these structures which might be the signatures 
of small areas of emission in the pulsar magnetosphere. It has to be noted that this can hardly be done in radio due to the faintness of the pulsar in this energy range (Manchester et al, 1993).

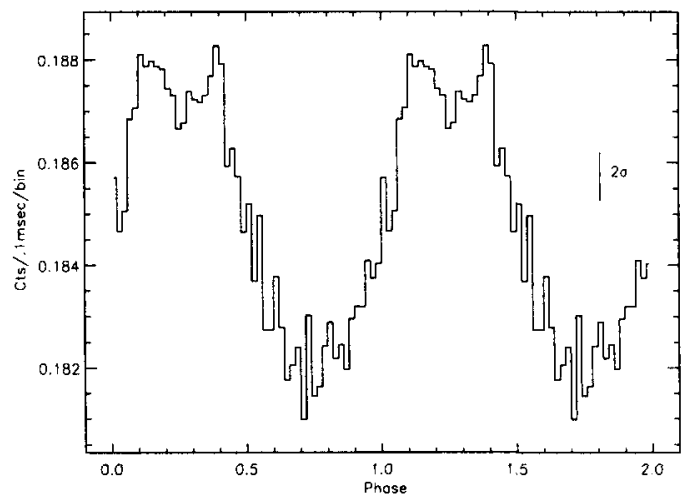

Figure 1. The Optical pulse shape from PSR0540-69 on 1992, November 5. Two full cycles are plotted.

PSR0540-69 was observed in soft X-rays band (0.1-2.4 KeV) with the PSPC detector on board the ROSAT satellite in 1991, February (Finley et al, 1993). We have a nearly contemporaneous optical observation (1991, January 10) which has been analyzed using the timing solution given by the ROSAT data. We could thus compare the two pulse profiles. Considering the different accuracies (an assumed 2 msec accuracy of the ROSAT clock with respect to the UTC), one can consider that the X-ray pulse and the optical pulse are in phase. Again narrow features in the pulse shape are visible in both energy ranges. However higher signal to noise light curves are required to refine such correlations.

Acknowledgments. This work was based on observations obtained at the European Southern Observatory, La Silla, Chile. The authors thank the support of the ESO technical staff at the observatory.

\section{References}

Boyd et al 1995, ApJ, 448, 365

Buccheri, R. et al 1983, A\&A, 128, 245

Finley et al 1993, ApJ, 410, 323

Gouiffes, C., Finley, J.P. and Ögelman, H. 1992, Ap.J, 394, 581

Manchester et al 1993, ApJ, 403, L29

Middleditch, J. and Pennypacker, C.R. 1985, Nature, 313, 659

Seward et al 1984 ApJ, 287, L19 


\section{The Polarization of the Crab Pulsar with HST}

Joseph F. Dolan, Patricia T. Boyd ${ }^{*}$ and Robert J. Hill ${ }^{+}$

Laboratory for Astronomy and Solar Physics, NASA Goddard Space

Flight Center, Greenbelt, MD 20771

* also Universities Space Research Association

+ also Hughes STX Corporation

F. Graham-Smith and A. G. Lyne

University of Manchester, Nuffield Radio Astronomy Laboratories, Jodrell Bank, Macclesfield, Cheshire SK119DL, UK

James D. Biggs

Perth Observatory, Walnut Road, Bickley 6076, Western Australia

J. W. Percival

Department of Astronomy and Space Astronomy Laboratory, University of Wisconsin, Madison, WI 53706

Edward L. Robinson

Department of Astronomy and McDonald Observatory, University of Texas, Austin, TX 78712

G. Wayne van Citters

Division of Astronomical Sciences, National Science Foundation, 4201 Wilson Blvd., Arlington, VA 22230

The linear polarization of the Crab pulsar as a function of pulse phase was observed by the High Speed Photometer on the Hubble Space Telescope in March, 1993. Observations were obtained in a bandpass centered on 2770 A using a $0.25 \mathrm{~ms}$ sample time, corresponding to a time resolution of 0.0075 in pulse phase. The UV polarization of the pulsar [Fig. 1] is strikingly similar to that observed in the visible (cf. Smith et al. 1988). The same values of polarization and the same swing of position angle occur through the main and secondary pulses. The polarization pulse profile must be essentially wavelength independent at frequencies above the infrared.

Any model of the emission regions in the Crab pulsar must then be wavelength independent. Our observations support the geometrical model proposed by Smith et al. (1988), which ascribes the double pulse to radiation from regions above the two magnetic poles, and places them at a radial distance of about nine-tenths of the velocity-of-light radius. 